\title{
OBSERVATIONS OF THE BLAZAR 3C 66A WITH THE MAGIC TELESCOPES IN STEREOSCOPIC MODE
}

J. Aleksić ${ }^{1}$, L. A. Antonelli ${ }^{2}$, P. Antoranz ${ }^{3}$, M. Backes ${ }^{4}$, J. A. Barrio ${ }^{5}$, D. Bastieri ${ }^{6}$, J. Becerra González ${ }^{7,8}$, W. BednareK ${ }^{9}$, A. Berdyugin ${ }^{10}$, K. Berger ${ }^{7}$, E. Bernardini ${ }^{11}$, A. Biland ${ }^{12}$, O. Blanch ${ }^{1}$, R. K. Bock ${ }^{13}$, A. Boller ${ }^{12}$, G. Bonnoli ${ }^{2}$, P. Bordas ${ }^{14}$, D. Borla Tridon ${ }^{13}$, V. Bosch-Ramon ${ }^{14}$, D. Bose ${ }^{5}$, I. Braun ${ }^{12}$, T. Bretz ${ }^{15}$, M. Camara ${ }^{5}$, A. Cañellas ${ }^{14}$, E. Carmona ${ }^{13}$, A. Carosi ${ }^{2}$, P. Colin ${ }^{13}$, E. Colombo ${ }^{7}$, J. L. Contreras ${ }^{5}$, J. Cortina ${ }^{1}$, L. Cossio ${ }^{16}$, S. Covino $^{2}$, F. Dazzi ${ }^{16,25}$, A. De Angelis ${ }^{16}$, E. De Cea del Pozo ${ }^{17}$, B. De Lotto ${ }^{16}$, M. De Maria ${ }^{16}$, F. De Sabata ${ }^{16}$,

C. Delgado Mendez ${ }^{7,26}$, A. Diago Ortega ${ }^{7,8}$, M. Doert $^{4}$, A. Domínguez ${ }^{18}$, D. Dominis Prester ${ }^{19}$, D. Dorner ${ }^{12}$, M. Doro $^{6}$,

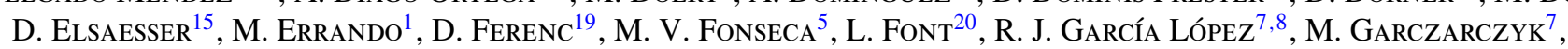
G. GiavitTo $^{1}$, N. Godinović ${ }^{19}$, D. Hadasch ${ }^{17}$, A. Herrero ${ }^{7,8}$, D. Hildebrand ${ }^{12}$, D. HöHNe-MöNCh ${ }^{15}$, J. Hose ${ }^{13}$, D. HruPeC ${ }^{19}$,

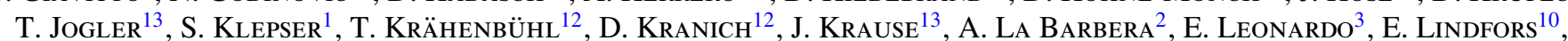

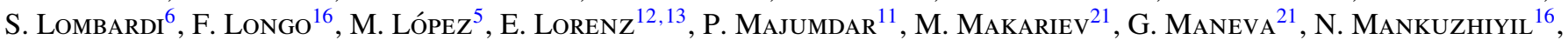
K. Mannheim ${ }^{15}$, L. Maraschi ${ }^{2}$, M. Mariotti ${ }^{6}$, M. Martínez ${ }^{1}$, D. Mazin ${ }^{1}$, M. Meucci ${ }^{3}$, J. M. Miranda ${ }^{3}$, R. Mirzoyan ${ }^{13}$, H. Miyamoto ${ }^{13}$, J. Moldón ${ }^{14}$, A. Moralejo ${ }^{1}$, D. Nieto ${ }^{5}$, K. Nilsson ${ }^{10,27}$, R. Orito ${ }^{13}$, I. Oya ${ }^{5}$, R. Paoletti ${ }^{3}$, J. M. Paredes ${ }^{14}$,

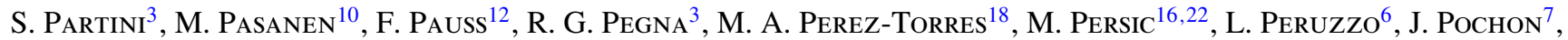
F. Prada ${ }^{18}$, P. G. Prada Moroni ${ }^{3}$, E. Prandini ${ }^{6}$, N. Puchades ${ }^{1}$, I. PuljaK ${ }^{19}$, I. Reichardt ${ }^{1}$, R. Reinthal ${ }^{10}$, W. Rhode ${ }^{4}$, M. Ribó ${ }^{14}$, J. Rico ${ }^{23,1}$, S. RÜGamer ${ }^{15}$, A. SagGion ${ }^{6}$, K. Saito ${ }^{13}$, T. Y. Saito ${ }^{13}$, M. Salvati ${ }^{2}$, M. Sánchez-Conde ${ }^{7,8}$, K. Satalecka ${ }^{11}$, V. Scalzotto ${ }^{6}$, V. Scapin ${ }^{16}$, C. Schultz ${ }^{6}$, T. Schweizer ${ }^{13}$, M. Shayduk ${ }^{13}$, S. N. Shore ${ }^{24}$, A. Sierpowska-BartosiK ${ }^{9}$, A. Sillanpä̈̈ ${ }^{10}$, J. Sitarek ${ }^{13,9}$, D. Sobczynska ${ }^{9}$, F. Spanier ${ }^{15}$, S. Spiro ${ }^{2}$, A. Stamerra ${ }^{3}$, B. Steinke ${ }^{13}$, J. Storz ${ }^{15}$, N. Strah ${ }^{4}$, J. C. Struebig ${ }^{15}$, T. Suric ${ }^{19}$, L. Takalo ${ }^{10}$, F. Tavecchio ${ }^{2}$, P. Temnikov ${ }^{21}$, T. Terzić ${ }^{19}$, D. Tescaro ${ }^{1}$, M. Teshima ${ }^{13}$, M. Thom ${ }^{4}$, D. F. Torres ${ }^{23,17}$, H. Vankov ${ }^{21}$, R. M. Wagner ${ }^{13}$, Q. Weitzel ${ }^{12}$, V. Zabalza ${ }^{14}$, F. ZANDANEL ${ }^{18}$, AND R. ZANIN ${ }^{1}$

${ }^{1}$ IFAE, Edifici Cn., Campus UAB, E-08193 Bellaterra, Spain; klepser@ifae.es

${ }_{2}^{2}$ INAF National Institute for Astrophysics, I-00136 Rome, Italy

${ }^{3}$ Università di Siena, and INFN Pisa, I-53100 Siena, Italy

${ }^{4}$ Technische Universität Dortmund, D-44221 Dortmund, Germany

${ }^{5}$ Universidad Complutense, E-28040 Madrid, Spain

${ }^{6}$ Università di Padova and INFN, I-35131 Padova, Italy

${ }^{7}$ Institute de Astrofísica de Canarias, E-38200 La Laguna, Tenerife, Spain

${ }^{8}$ Depto. de Astrofísica, Universidad de La Laguna, E-38206 La Laguna, Spain ${ }^{9}$ University of Łódź, PL-90236 Lodz, Poland

${ }^{10}$ Tuorla Observatory, University of Turku, FI-21500 Piikkiö, Finland

${ }^{11}$ Deutsches Elektronen-Synchrotron (DESY), D-15738 Zeuthen, Germany ${ }^{12}$ ETH Zurich, CH-8093, Switzerland

${ }^{13}$ Max-Planck-Institut für Physik, D-80805 München, Germany; ksaito@mppmu.mpg.de

${ }^{14}$ Universitat de Barcelona (ICC/IEEC), E-08028 Barcelona, Spain

${ }^{15}$ Universität Würzburg, D-97074 Würzburg, Germany

${ }^{16}$ Università di Udine, and INFN Trieste, I-33100 Udine, Italy

${ }^{17}$ Institut de Ciències de l'Espai (IEEC-CSIC), E-08193 Bellaterra, Spain

${ }^{18}$ Institute de Astrofísica de Andalucía (CSIC), E-18080 Granada, Spain

${ }^{19}$ Croatian MAGIC Consortium, Institute R. Boskovic, University of Rijeka and University of Split, HR-10000 Zagreb, Croatia

${ }^{20}$ Universitat Autònoma de Barcelona, E-08193 Bellaterra, Spain

${ }^{21}$ Institute for Nucl. Research and Nucl. Energy, BG-1784 Sofia, Bulgaria

${ }^{22}$ INAF/Osservatorio Astronomico and INFN, I-34143 Trieste, Italy

${ }^{23}$ ICREA, E-08010 Barcelona, Spain

${ }^{24}$ Università di Pisa, and INFN Pisa, I-56126 Pisa, Italy

Received 2010 November 1; accepted 2010 November 8; published 2010 December 15

\section{ABSTRACT}

We report new observations of the intermediate-frequency peaked BL Lacertae object 3C 66A with the MAGIC telescopes. The data sample we use were taken in 2009 December and 2010 January, and comprises $2.3 \mathrm{hr}$ of good quality data in stereoscopic mode. In this period, we find a significant signal from the direction of the blazar 3C 66A. The new MAGIC stereoscopic system is shown to play an essential role for the separation between $3 \mathrm{C} 66 \mathrm{~A}$ and the nearby radio galaxy $3 \mathrm{C} 66 \mathrm{~B}$, which is at a distance of only $6{ }^{\prime}$. The derived integral flux above $100 \mathrm{GeV}$ is $8.3 \%$ of the Crab Nebula flux and the energy spectrum is reproduced by a power law of photon index $3.64 \pm 0.39_{\text {stat }} \pm 0.25_{\text {sys }}$. Within errors, this is compatible with the one derived by VERITAS in 2009. From the spectra corrected for absorption by the extragalactic background light, we only find small differences between the four models that we applied, and constrain the redshift of the blazar to $z<0.68$.

Key words: BL Lacertae objects: individual (3C 66A) - galaxies: active - gamma rays: galaxies 


\section{INTRODUCTION}

Blazars make up the majority of extragalactic sources of very high energy (VHE; $E>100 \mathrm{GeV}$ ) gamma rays. They are a subset of active galactic nuclei (AGNs), and consist of BL Lacertae (BL Lac) objects and flat-spectrum radio-loud quasars. The general framework for explaining gamma-ray emission is that they are produced by charged particles which are accelerated in a relativistic jet. These jets are powered by gas accretion into a central supermassive black hole and are perpendicular to the accretion disc. When the jet is directed towards us, the energy and flux of the gamma rays are boosted by the relativistic beaming effect (e.g., Blandford \& Rees 1978; Urry \& Padovani 1995).

Generally, the spectral energy distribution of AGNs can be described by two broad bumps. The lower energetic bump, at frequencies from radio to X-rays, is attributed to synchrotron emission from nonthermal relativistic electrons in the jet. The other bump, covering the X-ray to gamma-ray bands, could either be due to inverse Compton scattering of seed photons by the electrons (leptonic model, e.g., Maraschi et al. 1992; Dermer \& Schlickeiser 1993; Bloom \& Marscher 1996; Krawczynski 2004) or due to hadronic interactions (see, e.g., Mannheim 1993; Mücke \& Protheroe 2001; Mücke et al. 2003).

3C 66A was classified as a BL Lac object by Maccagni et al. (1987), based on its significant optical and X-ray variability. The synchrotron peak of this source is located between $10^{15}$ and $10^{16} \mathrm{~Hz}$ (Perri et al. 2003), therefore 3C 66A can also be classified as an intermediate-frequency peaked BL Lac object (IBL). The redshift of 3C 66A was determined to be $z=0.444$ by independent authors (Miller et al. 1978; Lanzetta et al. 1993). However, their measurements are based on the detection of one single line. Another observation of $3 \mathrm{C} 66 \mathrm{~A}$ at a different spectral range was reported by Finke et al. (2008), but no spectral feature was found, and a lower limit of the redshift was derived to be 0.096. For the marginally resolved host galaxy (Wurtz et al. 1996), a redshift of 0.321 was found. Recently, through the investigation of the Large Area Telescope (LAT), on board the Fermi Gamma-ray Space Telescope (Fermi) satellite, and VHE gamma-ray observations, upper limits for the redshift of $3 \mathrm{C} 66 \mathrm{~A}$ were derived: $z=0.44$ (Prandini et al. 2010; $2 \sigma$ confidence level) and $z=0.58$ (Yang \& Wang 2010).

Several gamma-ray observations of 3C 66A were performed since the 1990s. With the EGRET satellite, a GeV gammaray emission (3EG J0222+4253) was associated with 3C 66A (Hartman et al. 1999). However, due to the large EGRET point-spread function (PSF), the influence of the nearby pulsar PSR J0218+4232 could not be excluded (Kuiper et al. 2000). The Crimean Astrophysical Observatory claimed detections of $3 \mathrm{C} 66 \mathrm{~A}$ above $900 \mathrm{GeV}$ with an integral flux of $(3 \pm 1) \times 10^{-11} \mathrm{~cm}^{-2} \mathrm{~s}^{-1}$ (Stepanyan et al. 2002). Later observations by HEGRA and Whipple reported upper limits of $F(>630 \mathrm{GeV})<1.42 \times 10^{-11} \mathrm{~cm}^{-2} \mathrm{~s}^{-1}$ (Aharonian et al. 2000 ) and $F(>350 \mathrm{GeV})<0.59 \times 10^{-11} \mathrm{~cm}^{-2} \mathrm{~s}^{-1}$ (Horan et al. 2004), respectively. Additionally, the STACEE observation found a hint of a signal at a 2.2 significance level and derived upper limits of $<1.0 \times 10^{-11} \mathrm{~cm}^{-2} \mathrm{~s}^{-1}$ and $<1.8 \times 10^{-11} \mathrm{~cm}^{-2} \mathrm{~s}^{-1}$ for thresholds of $147 \mathrm{GeV}$ and $200 \mathrm{GeV}$, respectively (Bramel et al. 2005).

\footnotetext{
${ }^{25}$ Supported by INFN Padova, Italy.

${ }^{26}$ Now at Centro de Investigaciones Energéticas, Medioambientales y Tecnológicas, Madrid, Spain.

${ }^{27}$ Now at Finnish Centre for Astronomy with ESO (FINCA), Turku, Finland.
}

Recent VERITAS observations of 3C 66A taken from 2007 September to 2008 January and from 2008 September to 2008 November, for a total of $32.8 \mathrm{hr}$, resulted in a detection in VHE gamma rays (Acciari et al. 2009). The energy spectrum was derived with a photon index of $\Gamma=4.1 \pm 0.4_{\text {stat }} \pm 0.6_{\text {sys }}$. The integral flux of the VERITAS observations above $200 \mathrm{GeV}$ is $(1.3 \pm 0.1) \times 10^{-11} \mathrm{~cm}^{-2} \mathrm{~s}^{-1}$ ( $6 \%$ of the Crab Nebula flux)

3C 66A has been monitored by Fermi/LAT since 2008 August, covering the latter part of the VERITAS observation. According to Abdo et al. (2009), who reported the first 5.5 months of Fermi/LAT observations of 3C 66A, the blazar showed a significant flux variability (a factor of 5-6 between the highest and lowest fluxes). The derived energy spectrum with photon index of $\Gamma=1.98$ above $1 \mathrm{GeV}$, in combination with the VERITAS spectrum, indicates that the spectrum must soften above $100 \mathrm{GeV}$.

MAGIC observed the sky region around 3C 66A from 2007 August to December, obtaining a total exposure time after data quality cuts of $45.3 \mathrm{hr}$ (Aliu et al. 2009b). These data revealed a significant VHE gamma-ray signal centered at $22^{\mathrm{h}} 23^{\mathrm{m}} 12^{\mathrm{s}}$, $43^{\circ} 0^{\prime} 7^{\prime \prime}$. This excess (named MAGIC J0223+430) coincides within uncertainties with the position of a nearby, FanaroffRiley-I (FRI) type galaxy 3C 66B $(z=0.0215$; Stull et al. 1975). Still, judging from the skyplot alone, the probability of the emission to originate from $3 \mathrm{C} 66 \mathrm{~A}$ is $14.6 \%$. The energy spectrum of MAGIC J0223+430 was reproduced by a single power law with the index of $\Gamma=3.1 \pm 0.3$. The integral flux above $150 \mathrm{GeV}$ corresponded to $(7.3 \pm 1.5) \times 10^{-12} \mathrm{~cm}^{-2} \mathrm{~s}^{-1}$ (2.2\% of the Crab Nebula flux). According to Tavecchio \& Ghisellini (2008), the radio galaxy is also a plausible source of VHE gamma-ray radiation. Also, the recent MAGIC detection of IC 310 (Mariotti et al. 2010), a radio galaxy at a very similar redshift $(z=0.0189)$ indicates that $3 \mathrm{C} 66 \mathrm{~B}$ might feasibly explain all or part of the MAGIC detection from 2007.

\section{OBSERVATIONS}

From mid 2009 August, 3C 66A went into an optical high state, which was reported by the Tuorla blazar monitoring program. ${ }^{28}$ This outburst triggered new MAGIC observations. The optical flux in the $R$ band reached a maximum level of $\sim 12$ mJy in 2010 January, while the baseline flux in the historical data of the source is $\sim 6 \mathrm{mJy}$.

The observations were carried out with the MAGIC telescopes located on the Canary Island of La Palma $\left(28^{\circ} 8 \mathrm{~N}, 17.8\right.$ $\mathrm{W}, 2220 \mathrm{~m}$ a.s.1.). The two $17 \mathrm{~m}$ diameter telescopes use the atmospheric Cherenkov imaging technique and allow for measurements at a threshold as low as $50 \mathrm{GeV}$ in normal trigger mode.

We observed the blazar 3C 66A in several time slots between 2009 September and 2010 January. However, the sky imaging CCD cameras that are used to cross-check the telescope pointing ("starguider cameras") only became fully applicable to stereo observations in early December. To allow for a high-confidence directional statement on the arcminute scale, we therefore only used data taken after these upgrades, which were $5.6 \mathrm{hr}$ in total. Furthermore, we had to discard data with low event rates, affected by the exceptionally bad weather conditions in that winter. Finally, we had $2.3 \mathrm{hr}$ of good quality data left after all quality cuts. They were taken on six days between 2009 December 5 and 2010 January 18, partly under low-intensity moon light conditions.

\footnotetext{
28 http://users.utu.fi/kani/1m/index.html
} 
The data were taken using the false source tracking (wobble) method (Fomin et al. 1994), in which the pointing direction alternates every 20 minutes between two positions, offset by \pm 0.4 in RA from the source. These wobble positions were chosen with respect to $3 \mathrm{C} 66 \mathrm{~A}$, but the small distance to 3C 66B (0.01) allows equal judgment for both sources. The data were taken at zenith angles between $13^{\circ}$ and $35^{\circ}$.

\section{DATA ANALYSIS}

For the analysis, only stereoscopic events triggered by both MAGIC telescopes were used. They were analyzed in the MARS analysis framework (Moralejo et al. 2009), taking advantage both of the advanced single-telescope algorithms (e.g., Aliu et al. 2009a) and newly developed stereoscopic analysis routines. These routines are at present still subject to some minor improvements and will be discussed in more detail in a separate paper still in preparation, but are shortly outlined in the following.

Combining monoscopic and stereoscopic strategies, the direction of gamma rays is calculated for each telescope separately, using the random forest technique (Albert et al. 2008b), and later combined with the projected crossing point of the image axes, with a weight depending on the angle between the two shower images. Requiring a certain level of agreement between the different estimates furthermore improves the resolution, and also helps to reject the (less focused) hadron showers. Similarly, an energy estimator is determined from look-up tables for each telescope separately, and later combined to a common estimated energy.

The skymap generation, which is particularly important for the analysis of data from the $3 \mathrm{C} 66 \mathrm{~A} / \mathrm{B}$ region, follows a two-step algorithm. The first step is to generate an exposure model for the field of view in camera coordinates, for the quality cuts that were applied in the analysis. This is done by joining the distributions of photon-like events from the two wobble positions, taking advantage of the fact that the source, in relative camera coordinates, is on opposite sides for both wobble sets.

The second step is the calculation of an expected background event distribution in celestial coordinates, and its comparison to the actual event distribution. Before that comparison, a smearing with a Gaussian kernel is applied. The significances are calculated following Equation (17) of Li \& Ma (1983), taking into account the higher precision of the background estimation implied by the above modeling.

The performance of the analysis software was optimized and checked with contemporaneous Crab Nebula data and MC. The Crab Nebula spectrum could be analyzed down to about $50 \mathrm{GeV}$, fully covering the range of the spectrum presented in the next paragraph. The achieved angular resolution, defined as the $\sigma$ of a two-dimensional Gaussian function, is around 0.1 at $100 \mathrm{GeV}$ and approaching 0.065 at higher energies. This $\sigma$ defines the radius in which $39 \%$ of all photons of a point source are contained. The systematic uncertainty on the direction reconstruction is a product of the telescope pointing uncertainty and possible biases that occur in the reconstruction algorithms. The latter can be caused by irregularities in the shower images, such as missing camera pixels, inhomogeneous noise from stars in the field of view, or imperfections in the data acquisition electronics. Both the total pointing deviation and the telescope pointing precision of MAGIC were always monitored over the years (Bretz et al. 2009; Aleksić et al. 2010), and along with studies of contemporary stereo data of known direction lead

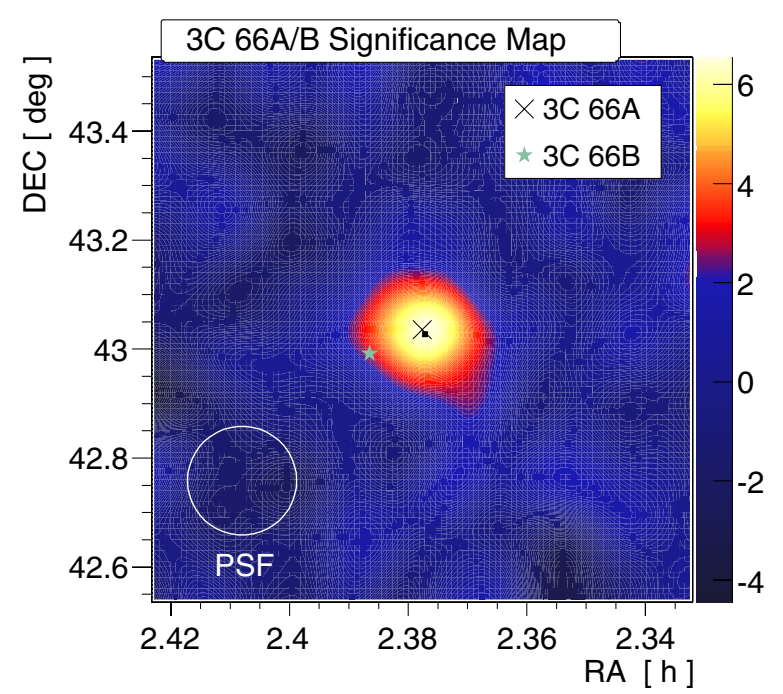

Figure 1. MAGIC significance skymap of the region around 3C 66A/B for events with energies above $100 \mathrm{GeV}$.

to an estimate of the maximal systematic stereoscopic pointing uncertainty of 0.025 .

We also used the publicly accessible Fermi/LAT data ${ }^{29}$ to investigate the status of the source in the $\mathrm{GeV}$ energy range during the MAGIC observation period. The Fermi data were analyzed using the public software package LAT Science Tools v9.15.2, including the Instrument Response File P6_V3_DIFFUSE, and galactic, extragalactic and instrumental background models.

\section{RESULTS}

Figure 1 shows a skymap of the observed region above $100 \mathrm{GeV}$. The significance of the excess at the location of $3 \mathrm{C} 66 \mathrm{~A}$ is $6.4 \sigma$. We also cross-checked the detection by investigating the distribution of squared angular distances $\left(\theta^{2}\right)$ between photon directions and the assumed source position. The expected background is extracted from corresponding $\theta^{2}$ plots done with respect to other sky positions at similar distances from the pointing direction. Comparing the data with this expectation we find a significance of $5.2 \sigma$ (see Figure 2). The difference in significance can be attributed to the different integration procedure of signal and background in the skymap, which generally leads to a slightly better background estimation and therefore a higher significance.

We also analyzed the data taken with and without moon light separately to find possible effects from the higher thresholds of individual camera pixels. However, we could not find a clear tendency beyond the statistical errors and thus decided to use all the data for the analysis.

Unlike in the 2007 observations of this sky region, the emission peak this time is clearly on top of $3 \mathrm{C} 66 \mathrm{~A}$. The fitted center of gravity of the excess (small black square in Figure 1) is at a distance of $0.010 \pm 0.023$ (stat.) \pm 0.025 (sys.) from 3C 66A, and $0.108 \pm 0.023$ (stat.) \pm 0.025 (sys.) from 3C 66B. While being compatible with the former, the statistical rejection power for the emission to emerge from the radio galaxy $3 \mathrm{C} 66 \mathrm{~B}$ corresponds to 4.6 standard deviations. Even considering the unlikely case of a systematic offset exactly toward the blazar, the rejection significance of $3 \mathrm{C} 66 \mathrm{~B}$ is at least $3.6 \sigma$. These numbers were confirmed by a second analysis with independent data

\footnotetext{
29 http://fermi.gsfc.nasa.gov/
} 


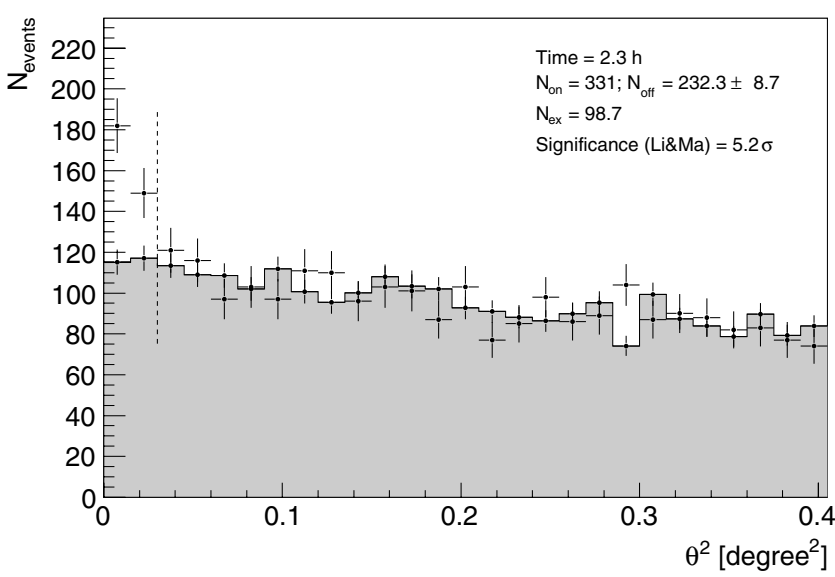

Figure 2. Distribution of squared angular distances between photon directions and the position of $3 \mathrm{C} 66 \mathrm{~A}\left(\theta^{2}\right)$ for events with energies above $100 \mathrm{GeV}$. The OFF data are taken from three positions that are symmetrical with respect to the telescope pointing directions.

quality selection and cut optimization procedures. The same result is found even when the photon direction is taken only from the projected crossing point of the two shower axes. We therefore conclude that the signal we see this time emerges from the blazar 3C 66A.

It shall be mentioned that this result is a clear merit of the angular resolution and background rejection of the new stereoscopic system. In fact, if we compare the above stereo directional reconstruction algorithm to the MAGIC-I algorithm alone, we basically find the same result, but the statistical error of the fitted source position increases roughly by a factor of two. Consequently, the rejection significance of $3 \mathrm{C} 66 \mathrm{~B}$ would be less than 2 standard deviations, and the total detection significance would be below 5 standard deviations.

The energy spectrum of 3C 66A was derived using four different unfolding algorithms (Albert et al. 2007) which correct for efficiency, smearing, and biasing effects in the energy response of the detector. The most conservative of these methods is the so-called forward unfolding, in which essentially a spectral shape is assumed a priori, and its parameters are adjusted by iteratively folding the assumed spectrum with the response function until the predicted distribution of estimated energies optimally matches the actually measured distribution. With all unfolding methods, we found that the data are well compatible with a power law of the form

$$
\frac{d F}{d E}=K_{200}\left(\frac{E}{200 \mathrm{GeV}}\right)^{-\Gamma},
$$

with a photon index $\Gamma=3.64 \pm 0.39_{\text {stat }} \pm 0.25_{\text {sys }}$ and a flux constant at $200 \mathrm{GeV}$ of $K_{200}=9.6 \pm 2.5_{\text {stat }} \pm 3.4_{\text {sys }} \times$ $10^{-11} \mathrm{~cm}^{-2} \mathrm{~s}^{-1} \mathrm{TeV}^{-1}$. The integral flux above $100 \mathrm{GeV}$ corresponds to $(4.5 \pm 1.1) \times 10^{-11} \mathrm{~cm}^{-2} \mathrm{~s}^{-1}(8.3 \%$ Crab Nebula flux). Here, the parameters and statistical errors are taken from the forward unfolding, while the systematic errors reflect the variations among the other unfolding algorithms, plus several standard uncertainties discussed in Albert et al. (2008a). The systematic flux uncertainties add up to $36 \%$ in total. Figure 3 displays the function we fitted through forward unfolding, and spectral points derived using the Tikhonov unfolding method (Tikhonov \& Arsenin 1979).

Due to the shortness of our observation, we cannot discuss flux variability with these data. However, comparing the flux to

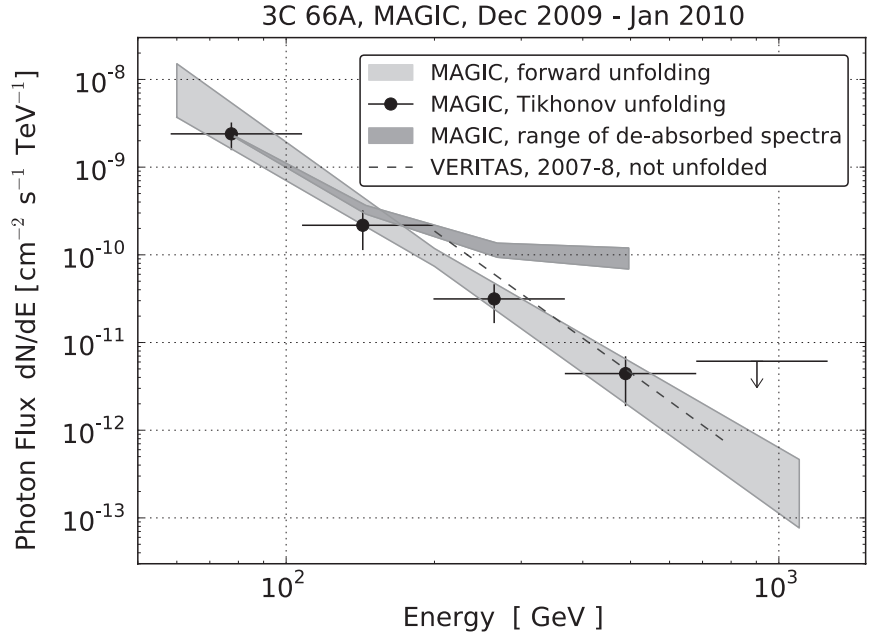

Figure 3. Observed and EBL-corrected (de-absorbed) differential energy spectra of 3C 66A in the period of 2009 December and 2010 January. The lightshaded area indicates the $1 \sigma$ range of the observed power-law spectrum gained by forward unfolding (see the text), the crosses are from the unfolding after Tikhonov \& Arsenin (1979) for comparison. The dark-shaded area is the spread of the de-absorbed, mean flux values obtained by the four applied EBL models, assuming the redshift of $z=0.444$. The VERITAS (observed) spectrum after Acciari et al. (2009) is shown for comparison.

the one from our previous observation of the $3 \mathrm{C} 66 \mathrm{~A} / \mathrm{B}$ region confirms the VERITAS report of $3 \mathrm{C} 66 \mathrm{~A}$ being a variable source in general.

We also analyzed the Fermi data from the same time period. The flux variability we found in a week-to-week light curve is not significant. Given the statistical uncertainties of the light curve, we would be sensitive on the $3 \sigma$ level to flux variations of $60 \%$ or greater, and conclude that the variability in the days we observed must be less than that. The averaged flux above $200 \mathrm{MeV}$ is roughly comparable to the averaged flux over the first 5.5 months (Abdo et al. 2009), and lower than that seen in 2008 October, when a strong TeV flare was observed by VERITAS. A single power-law model can reproduce the source spectrum, and the photon index is compatible with the one found in Abdo et al. (2009), indicating no significant change in the overall spectral shape.

\section{DISCUSSIONS AND CONCLUSIONS}

MAGIC observed the 3C 66A/B region in 2009 December and 2010 January, during an optical active state of 3C 66A and detected a clear VHE gamma-ray signal. The excess coincides with the position of $3 \mathrm{C} 66 \mathrm{~A}$, and we rule out the emission to come from $3 \mathrm{C} 66 \mathrm{~B}$ at a confidence level of $3.6 \sigma$. This detection does not contradict the earlier MAGIC detection, though, which favored $3 \mathrm{C} 66 \mathrm{~B}$ as the VHE source. On the one hand, because the observation time of $2.3 \mathrm{hr}$ would be too short to detect the VHE emission of $3 \mathrm{C}$ 66B, if on a similar flux level as in 2007 , and on the other hand, because its flux may be even lower than before. In fact, 3C 66A might have to be in a low flux state in order not to outshine the comparably weak emission from $3 \mathrm{C} 66 \mathrm{~B}$ at this close distance of about $1 \sigma$ of the PSF of the MAGIC telescopes.

The obtained energy spectrum is softer than in the previous MAGIC detection $\left(\Gamma=3.10 \pm 0.31_{\text {stat }} \pm 0.2_{\text {sys }}\right)$ and compatible with the VERITAS spectrum of 3C 66A. Compared to VERITAS, the MAGIC measurement has a lower threshold and the spectrum is extending to well below $100 \mathrm{GeV}$. The flux level of the $8.3 \%$ Crab Nebula flux is similar to the one reported by 
Table 1

EBL Corrected Indices

\begin{tabular}{lc}
\hline \hline \multicolumn{1}{c}{ Model } & $\Gamma_{\text {int }}$ \\
\hline Franceschini et al. (2008) & $2.57 \pm 0.68$ \\
Gilmore et al. (2009) & $2.61 \pm 0.67$ \\
Domínguez et al. (2010) & $2.59 \pm 0.68$ \\
Kneiske \& Dole (2010) & $2.37 \pm 0.70$
\end{tabular}

VERITAS (6\%), and significantly higher than in the previous MAGIC observation (2.2\%).

The VHE photons produced at the source can be absorbed in the intergalactic space by pair production with the low energy (UV to infrared) photons of extragalactic background light (EBL; Stecker et al. 1992; Hauser \& Dwek 2001). The amount of absorption depends on the energy and redshift, and can be corrected for in the data, assuming a certain modeling of the EBL density. Such a de-absorbed spectrum can be regarded as the spectrum we would measure if there were no EBL. To derive a de-absorbed spectrum, we tested several state of the art EBL models, namely, Franceschini et al. (2008), the fiducial model in Gilmore et al. (2009), Kneiske \& Dole (2010), and Domínguez et al. (2010). The EBL corrections were applied in the spectrum unfolding procedure (see above), using the full covariance matrix to correctly calculate the errors. The spread of the differential, de-absorbed flux spectra, obtained with the four models and assuming the redshift of $z=0.444$, is shown as the dark shaded area in Figure 3. The de-absorbed photon indices for the four EBL modelings are listed in Table 1. The differences between the de-absorbed spectra are very small, although the one corrected after Kneiske \& Dole (2010) is slightly harder than the others. This also reflects the fact that the predicted EBL shapes and densities are very similar in the first three models, but the overall density in Kneiske \& Dole (2010) is somewhat higher.

From most VHE emission models, the de-absorbed spectrum is expected not to be concave, i.e., rising toward higher energies. This can be tested both by comparing the points of our own spectrum, but also by a comparison with the Fermi photon index (1.98). The fact that we find our spectrum neither significantly concave nor harder than in Fermi suggests that the assumed redshift of $z=0.444$ does not contradict our observations. In fact, we investigated the plausibility of the redshift, assuming that the intrinsic spectrum is not expected to be exponentially rising, and thus have a pileup, at highest energies. This common method was previously used and described, for example, in Mazin \& Goebel (2007); Mazin \& Raue (2007). Using the Franceschini et al. (2008) model and the likelihood ratio test between the "power law" and "power law + pile-up" hypotheses, as described in the reference, we derive an upper limit on the redshift of $z<0.68$.

The results derived in this paper demonstrate the advantages of the MAGIC stereoscopic system. Further MAGIC and other gamma-ray observations of this region can provide interesting information about the IBL type BL Lac object 3C 66A, and, during low-flux periods of that, also the FRI type galaxy $3 \mathrm{C}$ 66B.

We thank the Instituto de Astrofísica de Canarias for the excellent working conditions at the Observatorio del Roque de los Muchachos in La Palma. The support of the German BMBF and MPG, the Italian INFN, the Swiss National Fund
SNF, and the Spanish MICINN is gratefully acknowledged. This work was also supported by the Marie Curie program, by the CPAN CSD2007-00042 and MultiDark CSD2009-00064 projects of the Spanish Consolider-Ingenio 2010 programme, by grant DO02-353 of the Bulgarian NSF, by grant 127740 of the Academy of Finland, by the YIP of the Helmholtz Gemeinschaft, by the DFG Cluster of Excellence "Origin and Structure of the Universe," and by the Polish MNiSzW Grant N N203 390834. The Fermi data were obtained from the High Energy Astrophysics Science Archive Research Center (HEASARC), provided by NASA's Goddard Space Flight Center.

\section{REFERENCES}

Abdo, A. A., et al. 2009, ApJ, 707, 1310

Acciari, V. A., et al. 2009, ApJ, 693, L104

Aharonian, F., et al. 2000, A\&A, 353, 847

Albert, J., et al. 2007, Nucl. Instrum. Methods A, 583, 494

Albert, J., et al. 2008a, ApJ, 674, 1037

Albert, J., et al. 2008b, Nucl. Instrum. Methods A, 588, 424

Aleksić, J., et al. 2010, A\&A, 524, A77

Aliu, E., et al. 2009a, Astropart. Phys., 30, 293

Aliu, E., et al. 2009b, ApJ, 692, L29

Blandford, R. D., \& Rees, M. J. 1978, in Pittsburgh Conf. on BL Lac Objects, ed. A. M. Wolfe (Pittsburgh, PA: Univ. Pittsburgh), 328

Bloom, S. D., \& Marscher, A. P. 1996, ApJ, 461, 657

Bramel, D. A., et al. 2005, ApJ, 629, 108

Bretz, T., Dorner, D., Wagner, R. M., \& Sawallisch, P. 2009, Astropart. Phys., 31,92

Dermer, C. D., \& Schlickeiser, R. 1993, ApJ, 416, 458

Domínguez, A., et al. 2010, MNRAS, in press (arXiv:1007.1459)

Finke, J. D., Shields, J. C., Böttcher, M., \& Basu, S. 2008, A\&A, 477, 513

Fomin, V. P., Stepanian, A. A., Lamb, R. C., Lewis, D. A., Punch, M., \& Weekes, T. C. 1994, Astropart. Phys., 2, 137

Franceschini, A., Rodighiero, G., \& Vaccari, M. 2008, A\&A, 487, 837

Gilmore, R. C., Madau, P., Primack, J. R., Somerville, R. S., \& Haardt, F. 2009, MNRAS, 399, 1694

Hartman, R. C., et al. 1999, ApJS, 123, 79

Hauser, M. G., \& Dwek, E. 2001, ARA\&A, 39, 249

Horan, D., et al. 2004, ApJ, 603, 51

Kneiske, T. M., \& Dole, H. 2010, A\&A, 515, A19

Krawczynski, H. 2004, New Astron. Rev., 48, 367

Kuiper, L., Hermsen, W., Verbunt, F., Thompson, D. J., Stairs, I. H., Lyne, A. J., Strickman, M. S., \& Cusumano, G. 2000, A\&A, 359, 615

Lanzetta, K. M., Turnshek, D. A., \& Sandoval, J. 1993, ApJS, 84, 109

Li, T.P., \& Ma, Y.Q. 1983, ApJ, 272, 317

Maccagni, D., Garilli, B., Schild, R., \& Tarenghi, M. 1987, A\&A, 178, 21

Mannheim, K. 1993, A\&A, 269, 67

Maraschi, L., Ghisellini, G., \& Celotti, A. 1992, ApJ, 397, 5

Mariotti, M., et al. 2010, ATel, 2510

Mazin, D., \& Goebel, F. 2007, ApJ, 655, L13

Mazin, D., \& Raue, M. 2007, A\&A, 471, 439

Miller, J. S., French, H. B., \& Hawley, S. A. 1978, in Pittsburgh Conf. BL Lac Objects, ed. A. M. Wolfe (PA: Univ. Pittsburgh), 176

Moralejo, A., et al. 2009, in Proc. 31st ICRC (Łódź), 469 (arXiv:0907.0943)

Mücke, A., \& Protheroe, R. J. 2001, Astropart. Phys., 15, 121

Mücke, A., Protheroe, R. J., Engel, R., Rachen, J. P., \& Stanev, T. 2003, Astropart. Phys., 18, 593

Perri, M., et al. 2003, A\&A, 407, 453

Prandini, E., Bonnoli, G., Maraschi, L., Mariotti, M., \& Tavecchio, F. 2010, MNRAS, 405, L76

Stecker, F. W., de Jager, O. C., \& Salamon, M. H. 1992, ApJ, 390, L49

Stepanyan, A. A., Neshpor, Y. I., Andreeva, N. A., Kalekin, O. P., Zhogolev, N. A., Fomin, V. P., \& Shitov, V. G. 2002, Astron. Rep., 46, 634

Stull, M. A., Price, K. M., Daddario, L. R., Wernecke, S. J., Graf, W., \& Grebenkemper, C. J. 1975, AJ, 80, 559

Tavecchio, F., \& Ghisellini, G. 2008, MNRAS, 394, L131

Tikhonov, A. N., \& Arsenin, V. J. 1979, Methods for the Solution of Ill-posed Problems (Moscow: Nauka)

Urry, M., \& Padovani, P. 1995, PASP, 107, 803

Wurtz, R., Stocke, J. T., \& Yee, H. K. C. 1996, ApJS, 103, 109

Yang, J., \& Wang, J. 2010, PASJ, 62, L23 